\title{
Association between the location of transposed ovary and ovarian dose in patients with cervical cancer treated with postoperative pelvic radiotherapy
}

Xiao-juan Lv $v^{1,2,3}$, Xiao-long Cheng ${ }^{4}$, Ye-qiang Tu ${ }^{1,2,3}$, Ding-ding Yan ${ }^{1,2,3}$ and Qiu Tang ${ }^{1,2,3^{*}}$ (D)

\begin{abstract}
Background and purpose: How to protect the ovarian function during radiotherapy is uncertain. The purpose of this study was to explore the association between the location of the transposed ovary and the ovarian dose in patients with cervical cancer received radical hysterectomy, ovarian transposition, and postoperative pelvic radiotherapy.
\end{abstract}

Methods: A retrospective analysis was conducted of 150 young patients with cervical cancer who received radical hysterectomy, intraoperative ovarian transposition, and postoperative adjuvant radiotherapy in Zhejiang Cancer Hospital. Association between location of the transposed ovaries and ovarian dose was evaluated. The transposed position of ovaries with a satisfactory dose was explored using a receiver operator characteristic curve (ROC) analysis. Patients' ovarian function was followed up 3 months and 1 year after radiotherapy.

Results: A total of 32/214 (15\%) transposed ovaries were higher than the upper boundary of the planning target volume (PTV). The optimum cutoff value of $>1.12 \mathrm{~cm}$ above the iliac crest plane was significantly associated with ovaries above the upper PTV boundary. When the ovaries were below the upper boundary of PTV, the optimum cutoff value of transverse distance $>3.265 \mathrm{~cm}$ between the ovary and PTV was significantly associated with ovarian max dose $($ Dmax $) \leq 4 \mathrm{~Gy}$, and the optimum cutoff value of transverse distance $>2.391 \mathrm{~cm}$ was significantly associated with ovarian Dmax $\leq 5 \mathrm{~Gy}$. A total of 77 patients had received complete follow-up, and 56 patients (72.7\%) showed preserved ovarian function 1 year after radiotherapy, which was significantly increased compared with 3 months (44.2\%) after radiotherapy.

Conclusions: The location of transposed ovaries in patients with cervical cancer is significantly correlated with ovarian dose in adjuvant radiotherapy. We recommend transposition of ovaries $>1.12 \mathrm{~cm}$ higher than the iliac crest plane to obtain ovarian location above PTV. When the transposed ovary is below the upper boundary of PTV, ovarian Dmax $\leq 4 \mathrm{~Gy}$ may be obtained when the transverse distance between the ovary and PTV was $>3.265 \mathrm{~cm}$, and the ovarian Dmax $\leq 5$ Gy may be obtained when the transverse distance was $>2.391 \mathrm{~cm}$.

Keywords: Dose limit, Ovarian transposition, Ovarian function, Pelvic radiotherapy, Uterine cervical neoplasms

\footnotetext{
* Correspondence: tangqiu@zjcc.org.cn

'Institute of Cancer and Basic Medicine (ICBM), Chinese Academy of

Sciences, Hangzhou, China

2Department of Gynecologic Radiation Oncology, Cancer Hospital of the

University of Chinese Academy of Sciences, Hangzhou, China

Full list of author information is available at the end of the article
}

(C) The Author(s). 2019 Open Access This article is distributed under the terms of the Creative Commons Attribution 4.0 International License (http://creativecommons.org/licenses/by/4.0/), which permits unrestricted use, distribution, and reproduction in any medium, provided you give appropriate credit to the original author(s) and the source, provide a link to the Creative Commons license, and indicate if changes were made. The Creative Commons Public Domain Dedication waiver (http://creativecommons.org/publicdomain/zero/1.0/) applies to the data made available in this article, unless otherwise stated. 


\section{Background}

Cervical cancer is the most common tumor of the female reproductive system worldwide. Approximately $30-40 \%$ of cervical cancer occurs in young women, which is an increasing trend [1]. With the progress of social economy, education, and medical technology, the survival rate of cervical cancer has been increasing yearly, and the post-treatment quality of life of patients has attracted the attention of both doctors and patients [2]. Radiotherapy is one of the main treatments for cervical cancer. The ovary is an organ that is sensitive to radiotherapy, a low dose can cause irreversible damage of the ovarian endocrine function in young patients. And then early menopause of these patients occurred which is associated with perimenopausal syndrome, including hot flashes, night sweats, irritability, and a series of lipid metabolic abnormalities, osteoporosis, as well as the corresponding complications of cardiovascular and cerebrovascular diseases [3]. Therefor, preservation of ovarian endocrine function is important to quality of life for the young patients with cervical cancer.

A total of $0.19-1.3 \%$ of cervical squamous cell carcinomas metastasize to the ovary, and metastasis rate of adenocarcinoma is $1.4-8.2 \%$ [4-6]. Therefore, ovarian preservation in patients with cervical squamous cell carcinoma is recognized as safe and feasible, whereas in other pathological types it remains controversial. The ovary is located in the pelvis and is damaged by postoperative pelvic radiotherapy [7]. McCall et al. first described the ovary transposition out of the pelvic radiation fields as an effective method to avoid radiation injury of ovaries during postoperative radiotherapy in 1958 [8]. Subsequently, ovarian transposition has been increasingly common to protect ovarian function in young patients with early cervical squamous cell carcinoma $[5,6,9]$. However, several reports have indicated that only approximately $50 \%$ of the transposed ovarian function can be preserved for patients treated with pelvic radiotherapy, whereas ovarian failure has been observed in other patients $[5,10,11]$.

The preservation of the ovarian endocrine function in patients with cervical cancer after radiotherapy is directly associated with ovarian dose [12, 13]. The ovarian dose received in pelvic radiotherapy might be related to the position of the transposed ovary. However, few studies have explored the relationship between ovarian transposition and ovarian dose. Currently, whether the ovary needs to be transposed to successfully retain ovarian function is still uncertain. Therefore, we designed the present study to explore the association between the location of the transposed ovary and ovarian dose in patients with cervical cancer who underwent radical hysterectomy, ovarian transposition, and postoperative pelvic radiotherapy. Furthermore, we sought to predict the position of ovarian transposition to obtain a satisfactory radiation dose of the ovary in postoperative pelvic radiotherapy.

\section{Methods \\ Patients}

A retrospective analysis was conducted of 150 young patients with cervical cancer who underwent radical hysterectomy, intraoperative ovarian transposition and postoperative adjuvant radiotherapy in Zhejiang Cancer Hospital (Hangzhou, Zhejiang, China) from January 2011 to June 2017. This study was approved by the Ethics Committee of the Zhejiang Cancer Hospital and the subject gave informed consent. All cervical cancer patients received standard radical hysterectomy and unilateral or bilateral ovarian transposition at the peritoneum of the paracolic sulci. All patients were $\leq 45$ years old (the youngest was 22 years old), with an average age of $35.8 \pm 5.24$ years. According to the International Federation of Gynecology and Obstetrics (FIGO) staging criteria in 2009 [14], 77 (51.3\%) patients were stage IB1, 39 (26\%) stage IB2, $13(8.7 \%)$ stage IIA1, 13 (8.7\%) stage IIA2, six (4\%) stage IIB, and two (1.3\%) had residual recurrence. Most of the cases were squamous cell carcinoma (140 cases, 93.3\%), followed by adenocarcinoma (five cases, 3.3\%), adenosquamous carcinoma (four cases, $2.7 \%$ ), and neuroendocrine carcinoma (one case, $0.7 \%$ ). All patients received external pelvic radiotherapy, and two patients with residual recurrence received external pelvic and vaginal intracavitary radiotherapy. External pelvic radiotherapy included three-dimensional conformal radiotherapy (3D-CRT) and intensity-modulated radiotherapy (IMRT).

\section{Radiotherapy}

The body membrane was first established for CT simulation and treatment of each patient. The Philips BrillianceTM16 row CT simulation machine with large aperture was used to simulate the location of all patients with postoperative adjuvant external radiotherapy. CT images were acquired using 5-mm-thick contiguous slices with a radiotherapy $\mathrm{CT}$ simulator (Philips Brilliance Big Bore) and transferred to the treatment planning system (Philips Pinnacle ${ }^{3}$ TPS). The clinical target volume (CTV) covered the common iliac, external iliac, internal iliac, obturator, presacral and parametrial lymph node regions, and was delineated according to the guidelines defining the pelvic node CTV in external beam radiotherapy for uterine cervical cancer of radiation therapy oncology group (RTOG) [15]. The planned target volume (PTV) was generated by uniformly expanding the CTV boundary by $0.7 \mathrm{~cm}$ in three dimensions. Organs at risk (OAR) including bladder, rectum, bowel bag, spinal cord, bone marrow and ovaries were contoured according to the 


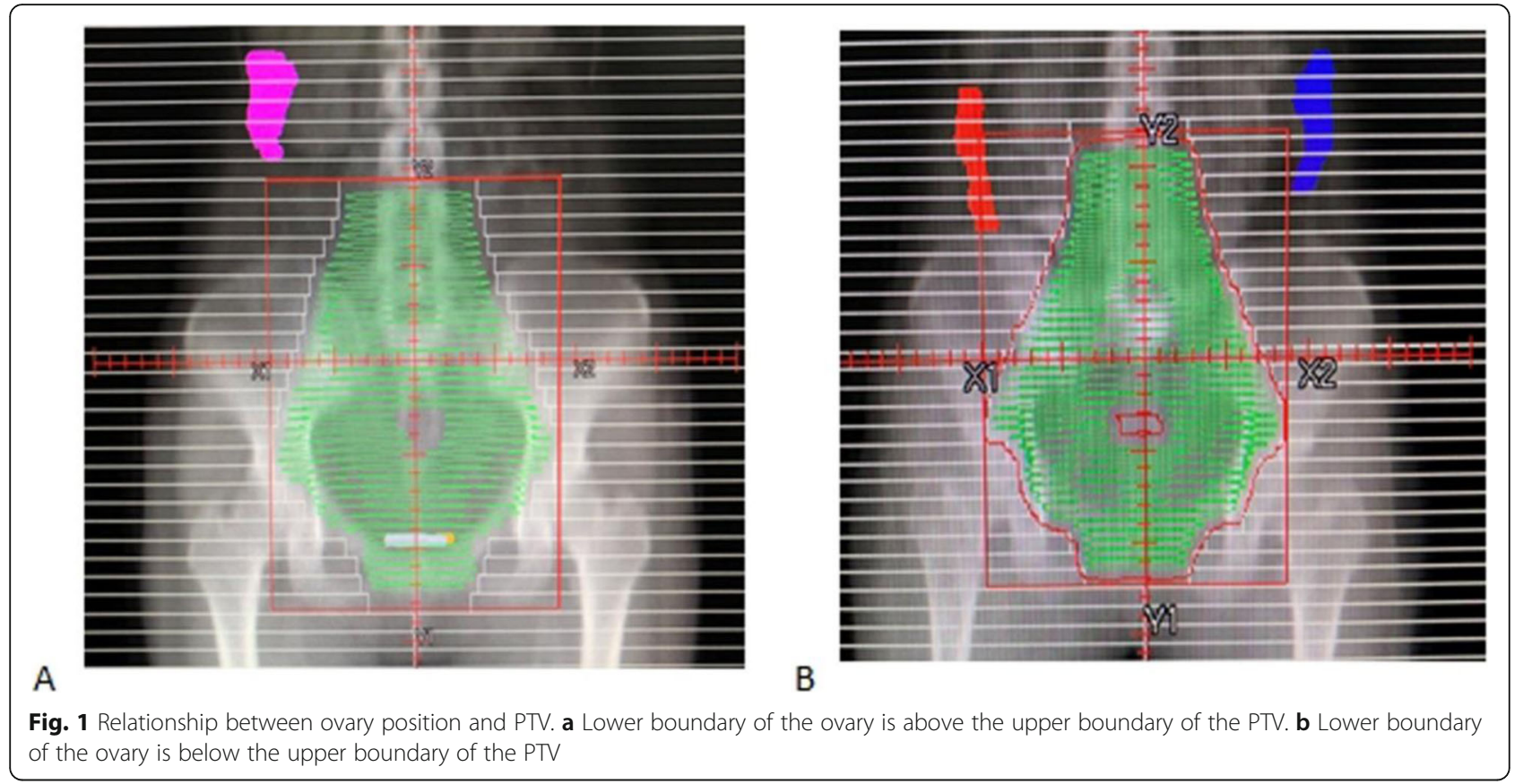

recommendations of the International Commission on Radiation Units Reports (ICRU) 50 and 62 [16, 17]. All radiotherapy plans were created by the physicists with the PTV prescription of 45-50Gy / 25-28F, and the Dmax of the ovaries was $\leq 6 \mathrm{~Gy}$. A total of $59 / 150$

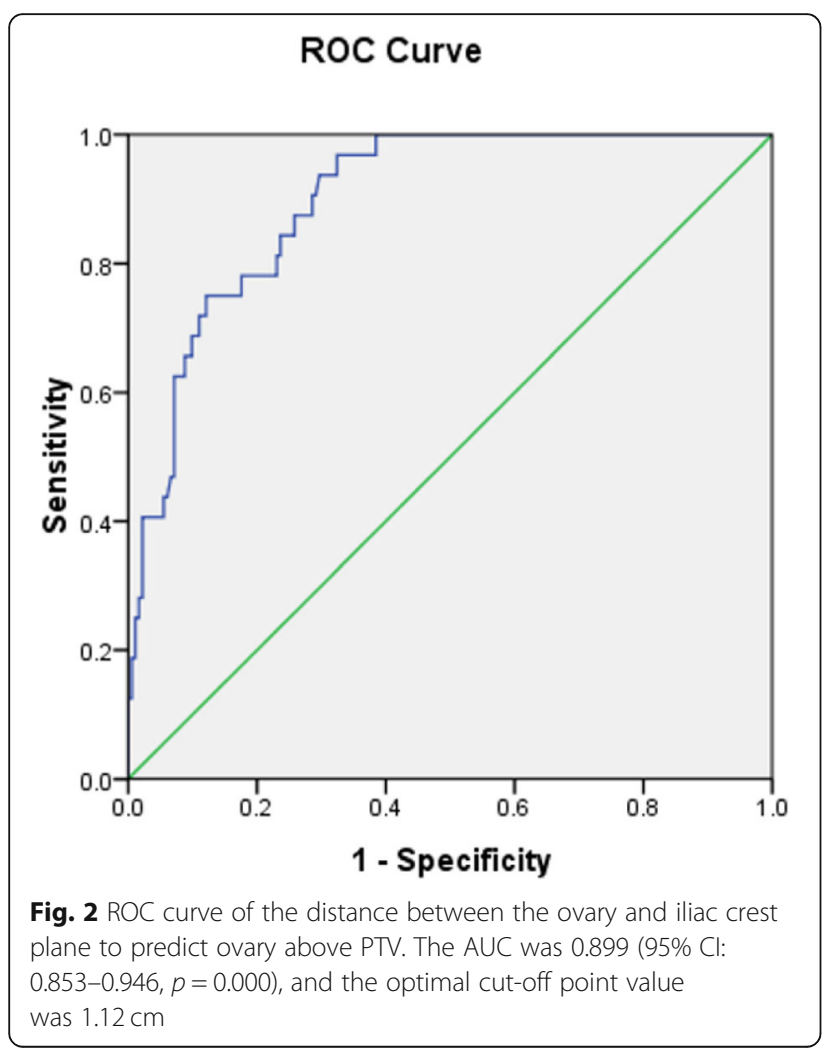

(39.3\%) patients received 3D-CRT, and 91/150 (60.7\%) patients received IMRT.

\section{Observations}

Records were reviewed for patients' age, height, weight, body mass index (BMI), clinical stage, pathological type, ovarian volume, ovarian cysts, side of ovarian transposition, method of radiotherapy, position of transposed ovary, and the Dmax of ovary. The position of the transposed ovary was measured as three distances including the vertical distance between the lower boundary of the ovary and the iliac crest plane, the vertical distance between the lower boundary of ovary and the upper boundary of the PTV, and the transverse distance between the medial boundary of the ovary and the lateral boundary of the PTV. The vertical distance was a negative value when the lower boundary of the ovary was below the iliac crest plane or below the upper boundary of the PTV. Based on the vertical distance between the lower boundary of the ovary and the upper boundary of the PTV, the patients were divided into two groups, including upper (the lower boundary of the ovary was above the upper boundary of the PTV) and lower (the lower boundary of the ovary was under the upper boundary of PTV) (Fig. 1). The observations of the two groups were compared and analyzed to explore the optimal location of the transposed ovary to be above the PTV. Ovaries which were partially or totally completely below the upper boundary of PTV and received dose limit were subdivided into two groups, including satisfactory (Dmax $\leq 400 \mathrm{cGy}$ ) and unsatisfactory (Dmax > $400 \mathrm{cGy})$. The observations of two groups were 
Table 1 Characteristics of patients with the ovary above or below PTV

\begin{tabular}{llll}
\hline Characteristics & Above PTV $(n=32)$ & Below PTV $(n=144)$ & $P$-value \\
\hline Ovarian volume, cm3 & $12.84 \pm 7.92$ & $13.63 \pm 9.13$ & $0.650^{*}$ \\
Ovarian cyst, case (rate) & $6(18.8 \%)$ & $29(20.1 \%)$ & $0.859^{\#}$ \\
Side of transposed ovaries & & $102(70.8 \%)$ & $0.117^{\#}$ \\
$\quad$ Right side & $27(84.4 \%)$ & $42(29.2 \%)$ & \\
$\quad$ Left side & $5(15.6 \%)$ & $47(32.6 \%)$ \\
Radiotherapy methods & & $97(67.4 \%)$ & $0.001^{\#}$ \\
3D-CRT & $21(65.6 \%)$ & $0.49 \pm 2.20$ & $0.000^{*}$ \\
IMRT & $11(34.4 \%)$ & $57(39.6 \%)$ & $0.000^{\#}$ \\
Distance from iliac crest plane, cm & $3.87 \pm 1.92$ & $32(100 \%)$ & \\
Dmax 400 cGy, case (rate) & &
\end{tabular}

"Student's $t$-test

${ }^{\#} x^{2}$ test

compared. The ROC curve was drawn to predict the location of the transposed ovary, where the ovary Dmax $\leq 400 \mathrm{cGy}$ and the ovary Dmax $\leq 500$ cGy could be obtained.

\section{Follow-up}

During the follow-up, the ovarian endocrine function of 150 patients were evaluated 3 months after radiotherapy and 1 year after radiotherapy. The sex hormone levels, including estrogen [E2], follicle stimulating hormone $[\mathrm{FSH}]$, and luteinizing hormone $[\mathrm{LH}]$ were measured.

Table 2 Characteristics of patients with the ovarian Dmax greater or less than 400 cGy

\begin{tabular}{|c|c|c|c|}
\hline Characteristics & $\begin{array}{l}\text { Dmax } \leq 400 \text { cGy } \\
(n=57)\end{array}$ & $\begin{array}{l}\text { Dmax }>400 \text { cGy } \\
(n=87)\end{array}$ & $\begin{array}{l}P \text { - } \\
\text { value }\end{array}$ \\
\hline Height, cm & $158.58 \pm 5.72$ & $159.29 \pm 4.47$ & $0.407^{*}$ \\
\hline Weight, kg & $56.15 \pm 10.69$ & $54.74 \pm 9.82$ & $0.638^{*}$ \\
\hline BMI & $22.06 \pm 3.85$ & $21.57 \pm 3.69$ & $0.445^{*}$ \\
\hline Ovarian volume, cm3 & $12.45 \pm 8.12$ & $14.40 \pm 9.69$ & $0.209^{*}$ \\
\hline Ovarian cyst, case (rate) & $11(19.3 \%)$ & $18(20.7 \%)$ & $0.839^{\#}$ \\
\hline $\begin{array}{l}\text { Side of transposed } \\
\text { ovaries }\end{array}$ & & & $0.083^{\#}$ \\
\hline Right side & $45(78.9 \%)$ & $57(65.5 \%)$ & \\
\hline Left side & $12(21.1 \%)$ & $30(34.5 \%)$ & \\
\hline Radiotherapy methods & & & $0.190^{\#}$ \\
\hline 3D-CRT & $15(26.3 \%)$ & $32(36.8 \%)$ & \\
\hline IMRT & $42(73.7 \%)$ & $55(63.2 \%)$ & \\
\hline $\begin{array}{l}\text { Distance from iliac crest } \\
\text { plane, cm }\end{array}$ & $1.08 \pm 2.28$ & $0.10 \pm 2.06$ & $0.008^{*}$ \\
\hline $\begin{array}{l}\text { Transverse distance from } \\
\text { PTV, cm }\end{array}$ & $4.03 \pm 1.15$ & $2.88 \pm 1.01$ & $0.000^{*}$ \\
\hline $\begin{array}{l}\text { Longitudinal distance } \\
\text { from PTV, cm }\end{array}$ & $-3.22 \pm 2.41$ & $-3.89 \pm 2.12$ & $.083^{*}$ \\
\hline
\end{tabular}

"Student's $t$-test

${ }^{\#} x^{2}$ test
Simultaneously, menopausal symptoms of the patients were assessed. Ovarian function was considered normal when the FSH was $<40 \mathrm{mIU} / \mathrm{mL}$ and E2 $>50 \mathrm{pg} / \mathrm{mL}$, and the patients showed no menopausal symptoms.

\section{Statistical analysis}

Mean values between the groups were compared using the Student's $t$ test. Frequency data between the groups were compared using the $\mathrm{X}^{2}$ test. A binary logistic regression model was used to determine the independent risk factors for satisfactory ovarian dose (yes vs. no). The receiver operating characteristic curve (ROC curve) was drawn to predict the location of the transposed ovary, where the ovary can above PTV and a satisfactory ovarian dose could be obtained. The data were analyzed by SPSS software (Statistical Package for the Social Sciences, SPSS, Version 19.0, Chicago, IL). A value of $P<$ 0.05 was considered statistically significant.

\section{Results}

A total number of 214 transposed ovaries were analyzed from 150 patients. Of the 150 patients, 78 (52\%) had a right ovarian transposition, 64 (42.7\%) had a bilateral ovarian transposition, and eight (5.3\%) had a left ovarian transposition. Out of the 214 transposed ovaries, there were $143(66.8 \%)$ on the right side and $71(33.2 \%)$ on the left side. The lower boundary of all transposed ovaries was $0.61 \pm 2.64 \mathrm{~cm}$ from the iliac crest plane. The lower boundary of the transposed ovary above the iliac crest plane was observed in $121(56.5 \%)$ cases, whereas the lower boundary of the ovary of $93(43.5 \%)$ cases was below the iliac crest plane. The distance between the lower boundary of the transposed ovary and the iliac crest plane showed a significant difference between the right and left ovaries $(1.05 \pm 2.64 \mathrm{~cm}$ vs. $-0.27 \pm 2.44 \mathrm{~cm}$, $t=3.551, P=0.000)$. There were $40 / 214(18.7 \%)$ ovarian cysts, and no ovarian metastases were observed. 
Table $\mathbf{3}$ Independent risk factors to predict the satisfactory ovarian dose of radiotherapy

\begin{tabular}{llll}
\hline Characteristics & OR & $95 \% C l$ & $P$-value \\
\hline Distance from iliac crest plane & 0.947 & $0.760-1.181$ & 0.631 \\
Transverse distance from PTV & 0.377 & $0.248-0.571$ & $0.000^{\&}$ \\
Longitudinal distance from PTV & 1.002 & $0.807-1.243$ & 0.985 \\
\hline${ }^{\&} P<0.05$ & & &
\end{tabular}

Out of the 214 transposed ovaries, 32 (15\%) were above PTV, and the Dmax was $\leq 400$ cGy, which was a satisfactory dose; 182 (85\%) transposed ovaries were partially or completely below the upper boundary of the PTV. Among these 182 ovaries, the radiation dose limit was abandoned in 38 because the ovaries were too close to the radiation fields, and the remaining 144 ovaries received the dose limit. We observed a significant difference in the distance between the ovary and the iliac crest plane, the radiotherapy method and the ovarian Dmax between the two groups $(P<0.05)$, as shown in Table 1 . The ROC curve was drawn, which is presented in Fig. 2. The distance between the transposed ovary and the iliac crest plane was significant for predicting the ovarian location above the PTV. The area under the curve [AUC] was 0.899 (95\% confidence interval [CI]: $0.853-0.946, P=0.000$ ). The optimal cut-off point value was $1.12 \mathrm{~cm}$, the sensitivity was 0.969 , the specificity was 0.676 , and the Youden index was 0.645.

A total of 144 ovaries which were partially or completely below the upper boundary of PTV and received dose limit were subdivided into two groups according to ovarian Dmax ( $\leq 400$ cGy or $>400$ cGy). Statistical analysis showed significant differences in the distances between the ovaries and the iliac crest plane as well as the transverse distance between the ovary and the PTV between the two Dmax level groups $(p<0.01)$, as can be seen in Table 2.

Logistic multivariate regression analysis showed that the transverse distance between the ovary and PTV was an independent correlative factor to predict the ovarian Dmax $\leq 400$ cGy during the radiotherapy, as shown in Table 3. The ROC curve was drawn, which is presented in Fig. 3. The transverse distance between the ovary and PTV was significant for predicting the ovarian Dmax $\leq 400$ cGy. The AUC was 0.779 (95\% CI: 0.703$0.856, p=0.000)$. The optimal cut-off point value was $3.265 \mathrm{~cm}$, the sensitivity was 0.807 , the specificity was 0.674 , and the Youden index was 0.481. The transverse distance between the ovary and PTV was also significant for predicting the satisfaction of ovarian Dmax $\leq 500$ cGy. The AUC was 0.755 (95\% CI: 0.638-0.872, $p=0.000$ ). The optimal cut-off point value was $2.391 \mathrm{~cm}$, the sensitivity was 0.832 , the specificity was 0.667 , and the Youden index was 0.499, as shown in Fig. 4.

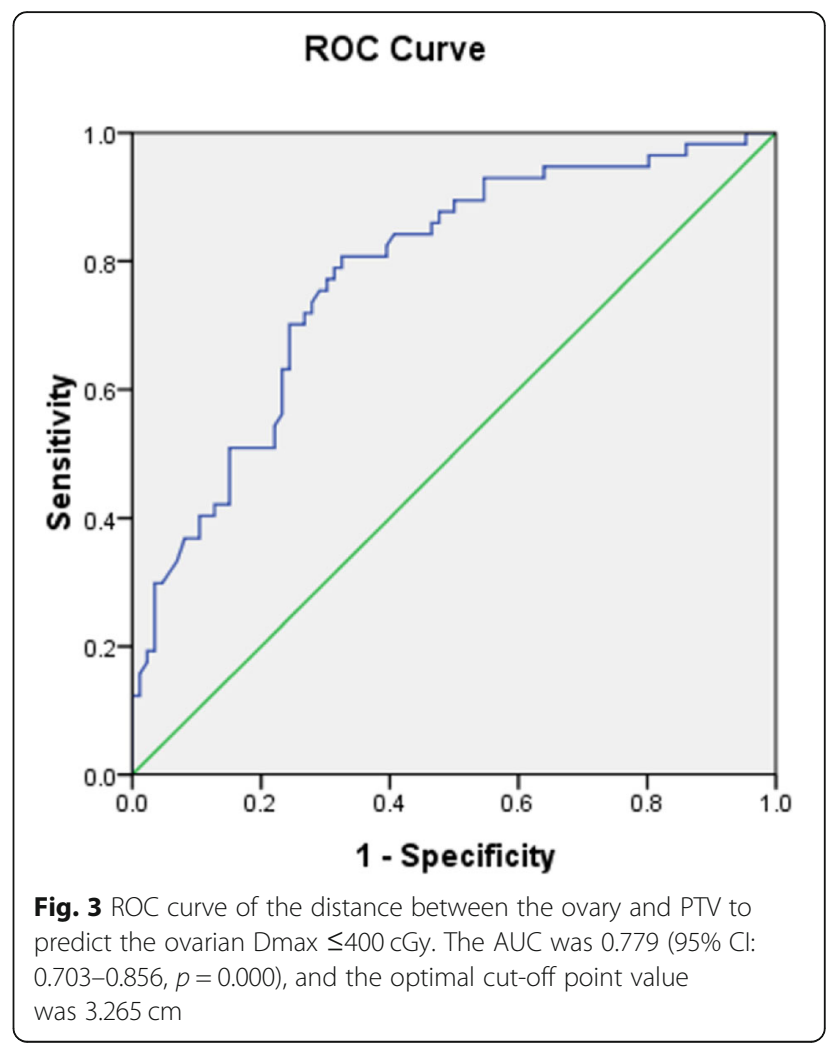

The ovarian endocrine function of 150 patients was evaluated 3 months and one-year post-radiotherapy, and $77 / 150$ patients reached complete followed-up. The sex hormones in the 77 patients were normal preradiotherapy. The E2 levels in the patients were significantly decreased 3 months post-radiotherapy, but significantly rebounded one-year post-radiotherapy. Conversely, the FSH and LH levels were significantly increased 3 months post-radiotherapy and were significantly decreased one-year post-radiotherapy, and the differences were statistically significant (Fig. 5, Table 4). According to FSH levels, E2 serum, and menopausal symptoms, 34/77 patients (44.2\%) had normal ovarian function 3 months post-radiotherapy. However, 7/34 patients lost ovarian function one-year post-radiotherapy, and 29 of the remaining 43 patients regained ovarian endocrine function one-year post-radiotherapy. Therefore, 56/77 (72.7\%) patients had preserved ovarian function one-year post-radiotherapy, which was significantly higher than 3 months post-radiotherapy $(p=0.000$; Table 4).

\section{Discussion}

Cervical cancer is a non-hormone dependent tumor, and the probability of early cervical cancer metastasis to the ovary is extremely low. Yamamoto et al. [18] reported ovarian metastasis in $2 / 485(0.4 \%)$ cases of early cervical squamous cell carcinoma and ovarian metastasis in 12/ 


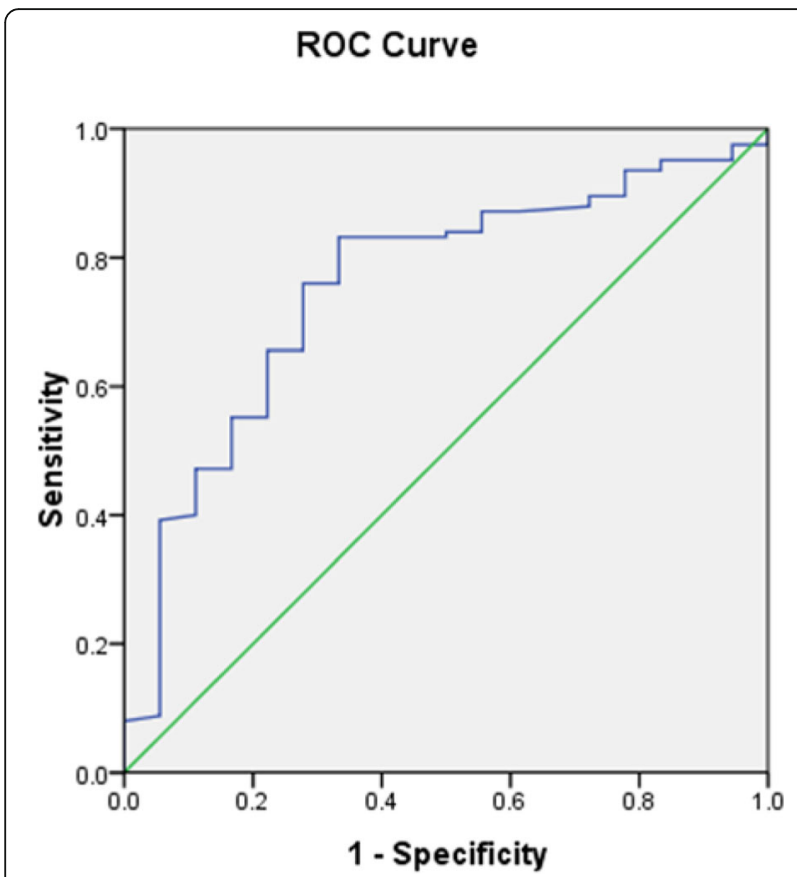

Fig. 4 ROC curve of the distance between the ovary and PTV to predict the ovarian Dmax $\leq 500$ cGy. The AUC was 0.755 (95\% Cl: $0.638-0.872, p=0.000$ ), and the optimal cut-off point value was $2.391 \mathrm{~cm}$
146 (8.2\%) cases of non-squamous cervical cancer. Additionally, Shimada et al. [19] found that ovarian metastasis in $52 / 3471(1.5 \%)$ patients with stage Ib-IIb cervical cancer, and that the metastasis rate of cervical adenocarcinoma was significantly higher than cervical squamous cell carcinoma (5.31\% vs. $0.79 \%)$. Landoni et al. [20] found ovarian metastasis in 16/1695 (0.9\%) cases of Ia2IIa cervical cancer with bilateral ovariotomy. Therefore, it is safe and feasible to retain ovaries in patients with early cervical squamous cell carcinoma. In the present study, the incidence of ovarian cysts was $18.7 \%$, and no case of translocated ovarian metastasis was found. In addition, the right ovary transposition was significantly higher than the left ovary, and the distance between the right ovary and the iliac crest plane was significantly higher than the left ovary. We speculated that this finding was related to the position of the surgeon, possibly the surgeon and staff were attending to the patient on the left side.

The rate of preserving the translocated ovarian endocrine function after pelvic radiotherapy was approximately $50 \%$, and the dose received by the ovary during radiotherapy and the age of the patients were two important influencing factors $[5,10,11]$. At the time of our study, there was no guideline or recommendation on the

\section{Sex hormone levels of patients}

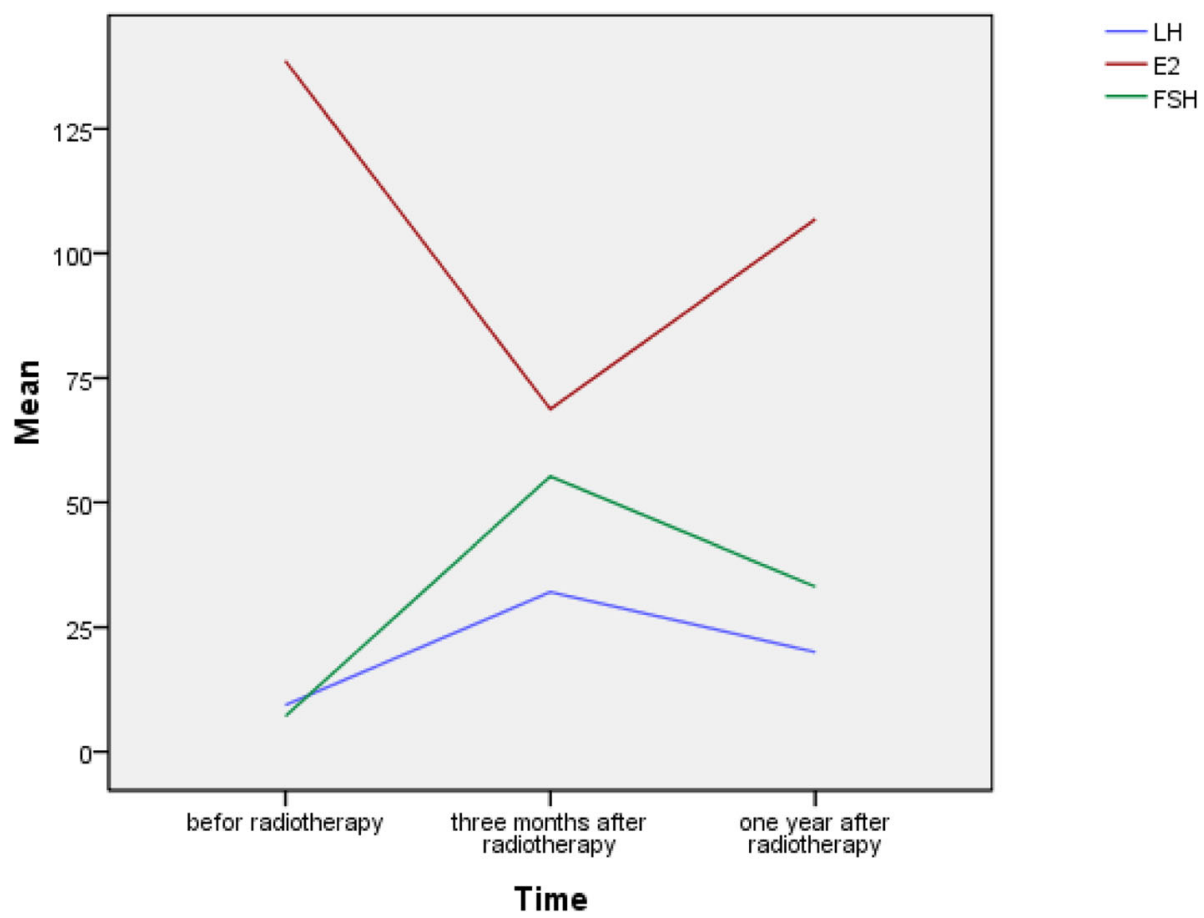

Fig. 5 Sex hormone levels at different times points pre- and post-radiotherapy. The E2 levels were significantly decreased 3 months postradiotherapy, but rebounded one-year post-radiotherapy. The FSH and LH levels were opposite trends 
Table 4 Sex hormone levels and ovarian function in patients pre- and post-radiotherapy

\begin{tabular}{llll}
\hline Characteristics & Pre-radiotherapy & Three months post-radiotherapy & One-year post-radiotherapy \\
\hline E2, pg/mL & $138.68 \pm 90.91$ & $68.75 \pm 98.77^{*}$ & $106.85 \pm 126.46^{\&}$ \\
FSH, $\mathrm{mlU} / \mathrm{mL}$ & $7.13 \pm 6.40$ & $55.25 \pm 43.35^{*}$ & $33.14 \pm 40.58^{\&}$ \\
$\mathrm{LH}, \mathrm{mlU} / \mathrm{mL}$ & $9.35 \pm 9.67$ & $32.09 \pm 24.49^{*}$ & $20.02 \pm 23.62^{\&}$ \\
Normal ovarian function, case (rate) & $77(100 \%)$ & $34(44.3 \%)$ & $56(72.7 \%)^{\#}$ \\
\hline
\end{tabular}

${ }^{*}$ Student's $t$-test, Comparison of sex hormone levels with pre-radiotherapy. $P<0.01$

${ }^{\&}$ Student's $t$-test, Comparison of sex hormone levels with 3 months post-radiotherapy. $P<0.05$

${ }^{\#} X^{2}$ test, Comparison of cases of normal ovarian function with 3 months post-radiotherapy. $P<0.01$

dose limit of radiotherapy in the ovary. Swerdlow et al. [21] found that the risk of premature ovarian failure for women under 40 years old was significantly increased when their ovaries received a radiation dose higher than 5 Gy. Husseinzadeh et al. [12] considered that the ovarian dose above $3 \mathrm{~Gy}$ significantly increased the risk of premature ovarian failure. Wallace et al. [13] found that a single dose of more than 8 Gy or a fractionated dose of more than $15 \mathrm{~Gy}$ caused a permanent damage of the ovarian function in most all women, whereas a dose lower than $1.5 \mathrm{~Gy}$ result in a minor effect on ovarian function. A follow-up study found that $64 \%$ of patients who received a radiation dose of less than 5 Gy after bilateral ovarian transposition retained their ovarian function [22]. Therefore, in our study, we considered a value of ovarian Dmax lower than 400 cGy to be a satisfactory dose.

The preservation of ovarian function has been directly related to its translocated position [23]. Charmber et al. [24] reported that $59-100 \%$ of patients would lost ovarian function when the ovary was transposed below the iliac crest, and $70-90 \%$ of patients would preserve the ovarian function when the transposed ovary above the iliac crest. Therefore, the ovary was recommended to be transposed over $1.5 \mathrm{~cm}$ above the iliac crest. Toman et al. [25] observed the endocrine function of transposed ovaries after external pelvic radiotherapy and found that $2.5 \mathrm{~cm}$ away from the edge of the radiation field was a safe area. Hwang et al. [9] reported that a distance greater than $1.5 \mathrm{~cm}$ between the transposed ovary and the iliac crest plane was an independent prognostic factor for the preservation of ovarian endocrine function. However there have been few studies reported to determine the relationship between the transposed position and the ovarian dose in radiotherapy. Yoshihiro et al. [26] used volumetric modulated arc therapy (VMAT) with limited angle to make plans and found that the average ovarian dose could be reduced to below $300 \mathrm{cGy}$ only when the transverse distance between the transposed ovaries and PTV was greater than $6.1 \mathrm{~cm}$.

In the present study, we found that the position of the transposed ovaries was significantly correlated with the ovarian dose in radiotherapy. When the transposed ovaries were above the PTV, a satisfactory dose could be obtained by IMRT or 3D-CRT. The ROC curve showed that the distance between the transposed ovaries and the iliac crest plane was significant for predicting the ovary above PTV, and the optimal cutoff value was $1.12 \mathrm{~cm}$. When the lower boundary of transposed ovaries below the upper boundary of the PTV, we found that the transverse distance between the ovary and PTV was significance for predicting the ovarian Dmax. For these patients, IMRT is recommended to reduce the ovarian radiation dose to a satisfactory level.

When the ovarian function is damaged by radiation, E2 secretion will be significantly reduced, whereas FSH and LH levels will increase, leading to perimenopausal symptoms, such as hot flashes and night sweats. The percentage of patients with normal ovarian function after radiotherapy varies widely. Buekers TE. et al. [11] analyzed 80 patients with cervical cancer who had received a radical hysterectomy and ovarian transposition and 26/80 received postoperative adjuvant radiotherapy, and found that only $42 \%$ of patients with postoperative adjuvant radiotherapy retained ovarian endocrine function at long-term follow-up. Pahisa J et al. [27] found that ovarian endocrine function was retained in $63.6 \%$ of patients with cervical cancer who received adjuvant radiotherapy after ovarian transposition, whereas ovarian function was retained in $93 \%$ of patients who did not receive adjuvant radiotherapy. AI-Badawi et al. [28] found that $65 \%$ of patients with cervical and rectal cancer who underwent ovarian transposition had successfully preserved ovarian endocrine function after a 33-month follow-up. Yin lina et al. [29] showed that 41/105 (39.0\%) patients who underwent IMRT with a limited radiation dose to the ovaries preserved their normal ovarian function. In the present study, 34 patients (44.2\%) exhibited normal ovarian function at 3 months after radiotherapy, however 56 patients (72.7\%) showed normal ovarian function at 1 year after radiotherapy. Interestingly, the average E2 level of the patients was the lowest 3 months after radiotherapy and gradually increased at one-year post-radiotherapy. Approximately $67 \%$ of patients who had lost ovarian endocrine function 3 months after radiotherapy regained it one-year postradiotherapy. This finding could be related to the different state of the follicles in the ovary, and the mature 
follicles are more sensitive to radiation than the primordial follicles [30]. When the ovaries receive low doses of radiation, the mature ovarian follicles with hormonesecreting function are first to die, whereas the primordial follicles survive. Following a long period of growth and development of into mature follicles, the ovary will likely secrete sex hormones again.

\section{Conclusions}

The location of the transposed ovary in patients with cervical cancer was significantly correlated with the ovarian dose of postoperative adjuvant radiotherapy. Transposition of the ovary higher than $1.12 \mathrm{~cm}$ above the iliac crest plane was recommended to obtain ovarian location above PTV. However, if the lower boundary of the transposed ovary is below the upper PTV boundary, the ovarian Dmax $\leq 400$ cGy may be obtained when the transverse distance between the ovary and PTV was > $3.265 \mathrm{~cm}$, and ovarian Dmax $\leq 5$ Gy may be obtained when the transverse distance was $>2.391 \mathrm{~cm}$ using IMRT.

\section{Abbreviations}

3D-CRT: Three dimensional conformal radiotherapy; AUC: Area under curve; Cl: Confidence interval; CTV: Clinical target volume; IMRT: Intensity modulated radiation therapy; OAR: Organ at risk; PTV: Planning target volume; ROC: Receiver operating characteristic curve; Se: Sensitivity; Sp: Specificity; Yl: Youden index

\section{Acknowledgments}

Not applicable.

\section{Authors' contributions}

XJL and QT conceived and designed the study. XLC interpreted the radiographic data. DDY and YQT interpreted the patients' clinical data. XJL was a major contributor in analyzing the data and writing the manuscript. QT reviewed the manuscript. All authors read and approved the final manuscript.

\section{Funding}

The study was supported by the Zhejiang Province Health Department Foundation, China (No.2018KY306)

\section{Availability of data and materials}

The datasets used and analyzed during the current study are available from the corresponding author on reasonable request.

\section{Ethics approval and consent to participate}

The present study was approved by the Ethic Committee of Zhejiang Cancer Hospital, and all participants gave written informed consent.

\section{Consent for publication}

Not applicable.

\section{Competing interests}

The authors declare that they have no competing interests.

\section{Author details}

${ }^{1}$ Institute of Cancer and Basic Medicine (ICBM), Chinese Academy of Sciences, Hangzhou, China. ${ }^{2}$ Department of Gynecologic Radiation Oncology, Cancer Hospital of the University of Chinese Academy of Sciences, Hangzhou, China. ${ }^{3}$ Department of Gynecologic Radiation Oncology, Zhejiang Cancer Hospital, Bangshan East Road 1, Hangzhou 310022, China. ${ }^{4}$ Department of Radiation Physics, Zhejiang Cancer Hospital, Hangzhou, China.
Received: 9 May 2019 Accepted: 6 December 2019

Published online: 16 December 2019

\section{References}

1. Bray F, Ferlay J, Soerjomataram I, Siegel RL, Torre LA, Jemal A. Global cancer statistics 2018: GLOBOCAN estimates of incidence and mortality worldwide for 36 cancers in 185 countries. CA Cancer J Clin. 2018;68(6):394-424.

2. Zeng H, Zheng R, Guo Y, Zhang S, Zou X, Wang N, et al. Cancer survival in China, 2003-2005: a population-based study. Int J Cancer. 2015;136(8):1921-30.

3. Kokcu A. Premature ovarian failure from current perspective. Gynecol Endocrinol. 2010;26:555-62.

4. Gubbala K, Laios A, Gallos I, Pathiraja P, Haldar K, Ind T. Outcomes of ovarian transposition in gynaecological cancers; a systematic review and metaanalysis. J Ovarian Res. 2014;7:69.

5. Xie X, Song K, Cui B, Jiang J, Yang X, Kong B. A comparison of the prognosis between adenocarcinoma and squamous cell carcinoma in stage IB-IIA cervical cancer. Int J Clin Oncol. 2018;23(3):522-31.

6. Matsuo K, Shimada M, Yamaguchi S, Kanao H, Nakanishi T, Saito T, et al. Identifying a candidate population for ovarian conservation in young women with clinical stage IB-IIB cervical cancer. Int J Cancer. 2018;142(5): 1022-32.

7. Turan AT, Keskin HL, Dundar B, Gundogdu B, Ozgul N, Boran N, et al. Ovarian transposition for stage lb squamous cell cervical cancer - lack of effects on survival rates? Asian Pac J Cancer Prev. 2013;14(1):133-7.

8. McCall ML, Keaty EC, Thompson JD. Conservation of ovarian tissue in the treatment of carcinoma of the cervix with radical surgery. Am J Obstet Gynecol. 1958;75:590-600.

9. Hwang JH, Yoo HJ, Park SH, Lim MC, Seo SS, Kang S, et al. Association between the location of transposed ovary and ovarian function in patients with uterine cervical cancer treated with (postoperative or primary) pelvic radiotherapy. Fertil Steril. 2012;97:1387-93.

10. Han SS, Kim YH, Lee SH, Kim GJ, Kim HJ, Kim JW, et al. Underuse of ovarian transposition in reproductive-aged cancer patients treated by primary or adjuvant pelvic irradiation. J Obstet Gynaecol Res. 2011;37:825-9.

11. Buekers TE, Anderson B, Sorosky JI. Ovarian function after surgical treatment for cervical cancer. Gynecol Oncol. 2001;80:85-8.

12. Husseinzadeh $\mathrm{N}$, van Aken ML, Aron B. Ovarian transposition in young patients with invasive cervical cancer receiving radiation therapy. Int J Gynecol Cancer. 1994:4:61-5.

13. Wallace WH, Shalet SM, Hendry JH, Morris-Jones PH. Gatta- maneni HR. ovarian failure following abdominal irradiation in childhood: the radiosensitivity of the human oocyte. Br J Radiol. 1989;62:995-8.

14. Pecorelli S. Revised FIGO staging for carcinoma of the vulva, cervix, and endometrium. Int J Gynaecol Obstet. 2009;105(2):103-4.

15. Small W Jr, Mell LK, Anderson P, Creutzberg C, De Los SJ, Gaffney D, et al. Consensus guidelines for delineation of clinical target volume for intensitymodulated pelvic radiotherapy in postoperative treatment of endometrial and cervical cancer. Int J Radiat Oncol Biol Phys. 2008;71(2):428-34.

16. International Commission on Radiation Units and Measurements. Prescribing, recording and reporting photon beam therapy Report No: ICRU Report 50. Bethesda, MD: ICRU; 1993.

17. International Commission on Radiation Units and Measurements. Prescribing, recording and reporting photon beam therapy (supplement to ICRU Report 50) Report No: ICRU Report 62. Bethesda, MD: ICRU; 1999.

18. Yamamoto R, Okamoto K, Yukiharu T, Kaneuchi M, Negishi H, Sakuragi N, et al. A study of risk factors for ovarian metastases in stage Ib-IIlb cervical carcinoma and analysis of ovarian function after a transposition. Gynecol Oncol. 2001;82:312-6.

19. Shimada M, Kigawa J, Nishimura R, Yamaguchi S, Kuzuya K, Nakanishi T, et al. Ovarian metastasis in carcinoma of the uterine cervix. Gynecol Oncol. 2006;101(2):234-7.

20. Landoni F, Zanagnolo V, Lovato-Diaz L, Maneo A, Rossi R, Gadducci A, et al. Ovarian metastases in early-stage cervical cancer (IA2-IIA): a multicenter retrospective study of 1965 patients (a cooperative task force study). Int J Gynecol Cancer. 2007;17(3):623-8.

21. Swerdlow AJ, Cooke R, Bates A, Cunningham D, Falk SJ, Gilson D, et al. Risk of premature menopause after treatment for Hodgkin's lymphoma. J Natl Cancer Inst. 2014;106(9):1-12. https://doi.org/10.1093/jnci/dju207.

22. Wallace $W H$, Thomson AB, Saran F, Kelsey TW. Predicting age of ovarian failure after radiation to a field that includes the ovaries. Int J Radiat Oncol Biol Phys. 2005;62:738-44. 
23. Yoon A, Lee YY, Park W, Huh SJ, Choi CH, Kim TJ, et al. Correlation between location of transposed ovary and function in cervical cancer patients who underwent radical hysterectomy. Int J Gynecol Cancer. 2015;25(4):688-93.

24. Chambers SK, Chambers JT, Kier R, Peschel RE. Sequelae of lateral ovarian transposition in irradiated cervical cancer patients. Int J Radiat Oncol Biol Phys. 1991;20(6):1305-8.

25. Toman J, Feyereisl J, Zámecník J, Frencl L. A safe site for transposition of ovaries in radical hysterectomy for cervical carcinoma. Ceska Gynekol. 2001; 66(3):184-6.

26. Yoshihiro U, Shingo O, Masaru I, Masayoshi M, Koji K, Shoji K, et al. Strategies for reducing ovarian dose in volumetric modulated arc therapy (VMAT) for postoperative uterine cervical cancer. Br J Radiol. 2018;91(1081): 20160777.

27. Pahisa J, Martínez-Román S, Martínez-Zamora MA, Torné A, Caparrós X, Sanjuán A, et al. Laparoscopic ovarian transposition in patients with early cervical cancer. Int J Gynecol Cancer. 2008;18(3):584-9.

28. Al-Badawi IA, Al-Aker M, AlSubhi J, Salem H, Abduljabbar A, Balaraj K, et al. Laparoscopic ovarian transposition before pelvic irradiation: a Saudi tertiary center experience. Int J Gynecol Cancer. 2010;20(6):1082-6.

29. Lina Y, Saiquan L, Jun Z, Zhang W, Ke G. Ovarian transposition before radiotherapy in cervical cancer patients: functional outcome and the adequate dose constraint. Radiat Oncol. 2019;14(1):100

30. Loren AW, Mangu PB, Beck LN, Brennan L, Magdalinski AJ, Partridge AH, et al. American Society of Clinical Oncology. Fertility preservation for patients with cancer: American Society of Clinical Oncology clinical practice guideline update. J Clin Oncol. 2013;31(19):2500-10.

\section{Publisher's Note}

Springer Nature remains neutral with regard to jurisdictional claims in published maps and institutional affiliations.

Ready to submit your research? Choose BMC and benefit from:

- fast, convenient online submission

- thorough peer review by experienced researchers in your field

- rapid publication on acceptance

- support for research data, including large and complex data types

- gold Open Access which fosters wider collaboration and increased citations

- maximum visibility for your research: over $100 \mathrm{M}$ website views per year

At $\mathrm{BMC}$, research is always in progress.

Learn more biomedcentral.com/submissions 